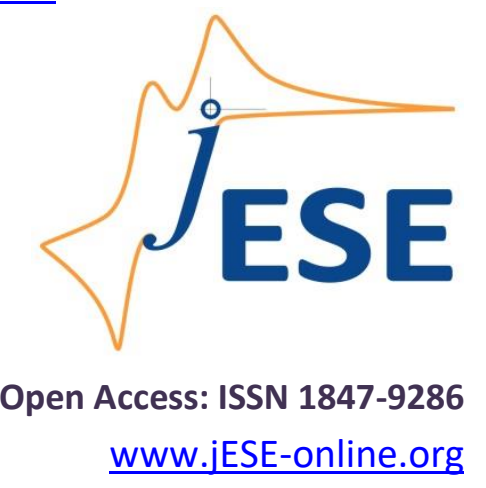

Original scientific paper

\title{
New electrochemical and physical measurements on sensitive glasses in thin-film technology
}

\author{
Frank Gerlach ${ }^{凶}$, Kristina Ahlborn, Winfried Vonau
}

Kurt-Schwabe-Institut für Mess- und Sensortechnik e.V. Meinsberg, 04736 Waldheim, Germany

Corresponding author: ${ }^{凶}$ frank.gerlach@ksi-meinsberg.de; Tel.: +49 34327608 116; Fax: +49 34327608131

Received: September 10, 2019; Accepted: October 7, 2019

\begin{abstract}
All-solid-state sensors have several advantages compared to conventional ones. These include, e.g., miniaturization, planar design, location independence and an uncomplicated realization of sensors for high pressure and high temperature. Therefore, a number of physical sensors, such as temperature sensors, pressure sensors, rotation angle sensors and force sensors are available in thin-film techniques. The presented paper shows the advantages to combination of thin and thick film techniques to manufacture electrochemical sensors, especially using pulsed laser ablation to create amorphous layers of glass. Furthermore, it investigates the range of possibilities for characterization of bulk and surface properties with electrochemical methods and specialized techniques with thermally stimulated currents (TSC).
\end{abstract}

\section{Keywords}

Amorphous glass, pulsed laser ablation, electrochemical impedance spectroscopy, $\mathrm{pH}$ sen-sor, redox sensor, thermally stimulated currents.

\section{Introduction}

Beside the advantages of all-solid-state sensors, it seems that special technological features lead to limited sensor capabilities because miniaturization and layer technologies reduce long-term stability and electrochemical performance compared to optical and conventional measurement technologies [1,2].

There are several demands of potential users for affordable, pressure-stable, low-service and low-maintenance, planar sensors. Generally, with screen printing techniques and vacuum technologies a wide range of electrochemical, glass-based sensors can be realized [3-10]. By using amorphous ion-sensitive glasses and their stable material properties, a good sensor behavior can be expected. The necessary reference systems, produced in this technology, can be executed as a combination of different ion-selective glasses [11]. This simplified arrangement is suitable for special applications in 
the process measurement technology and it provides stable measuring systems without disturbing outflow rates. Such glass-based all-solid-state multi-parameter sensor systems are used in expensive syntheses and in microreaction technology for the production of special pharmaceuticals. Although basing on the glass material, glass breakage and contamination are excluded and therefore such systems can be used in food industry without any problems.

State of the art are enamel systems measuring potential difference of two different sensitive electrodes [12]. An adequate measuring system offers by [13] as a double-membrane glass electrode. This was specially designed for applications in the chlor-alkali industry. The reference system to be used here is a pNa electrode which utilizes the stable sodium concentration in saline solution. An all-solid-state electrode likewise produced in thin-film technology is known as an ion-selective field effect transistor and is manufactured by means of classical semiconductor technology for example described by [14]. This manufacturing technology is very complex and focused on high volumes of sensors. To complete the measuring chain, a classical reference electrode is used here. In order to replace this in future measurements with an all-solid-state-system, it would be possible to carry out a differential measurement with a glass electrode reduced in terms of its parameterspecific sensitivity. This can minimize the systemic temperature dependencies and long-term drift by the symmetrical structure.

The focus of research article is on the one hand the development of sensitive, amorphous glass layers [15] and on the other hand, the development of a defined and reproducible transition of the ion-conducting glass layers to the electron-conducting noble metal conductive paths [16]. Since the chemical and electrochemical evaluation of the thin sensitive layers is very difficult, an attempt is made to extensively characterize the layer system using other integrative examination methods and thermal stimulations.

\section{Experimental}

\section{Development of sensitive glasses}

Ion-sensitive glasses are melted from powdery raw materials in high-temperature furnaces at $1200-1500{ }^{\circ} \mathrm{C}$. In order to receive geometrically clearly defined shapes, this still hot melt is poured into graphite molds, as shown in Figure 1. As a result, moldings (normally cylinders) are obtained. These are separated by means of a precision cutting saw with diamond grinding wheel (Accutom50, Struers, Willich (D)) into individual slices with a thickness of $5 \mathrm{~mm}$. Applied to a target holder, they can be used in different vacuum coating systems.
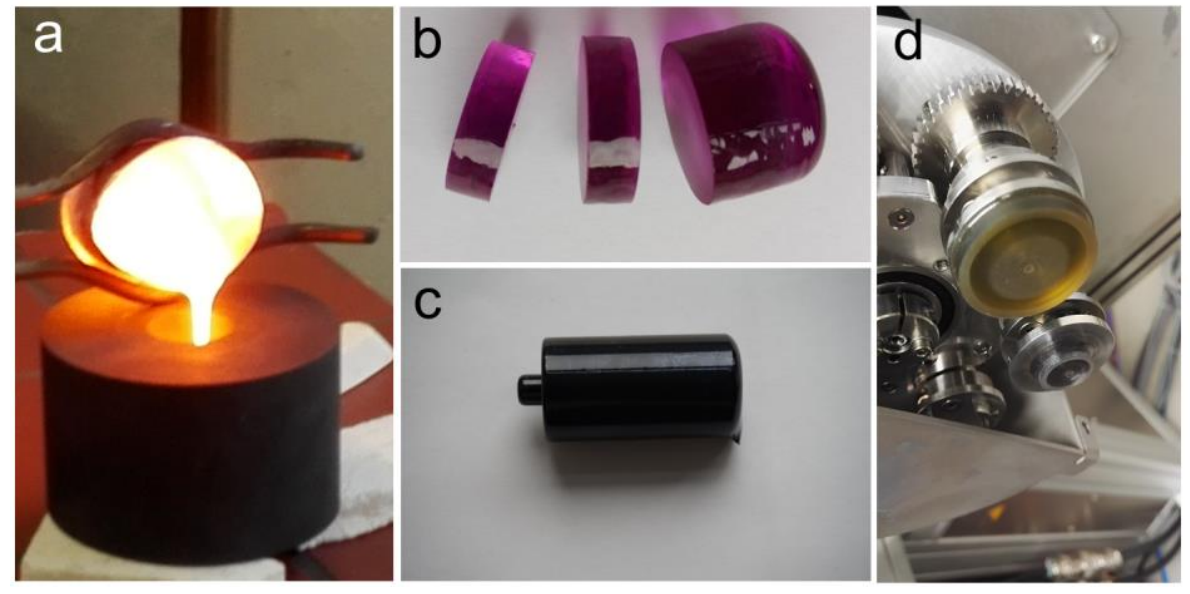

Figure 1. (a) glass melt in graphite molds. (b) sliced casting body. (c) molded body

(d) built in target at a target carousel 


\section{Fabrication of screen-printed chip structures}

Extensive screening studies require a large number of prototypes. The basic chip structures can be cost-effectively provided by screen printing technique in research projects. To minimize surface roughness, alumina substrates are coated with a glaze paste. These chips are equipped with a conductive path made of precious metal (gold or platinum), which are obtained from an organometallic paste, with a thickness of $500 \mathrm{~nm}$. Finally, with the exception of the sensory surface and the contacts, the whole assembly is hermetically sealed with glass. As part of the investigations, two different layouts were developed and used. Electrode structures, according to Figure 2, with a diameter of $7.5 \mathrm{~mm}$ were used for the standard morphological and electrochemical investigations.

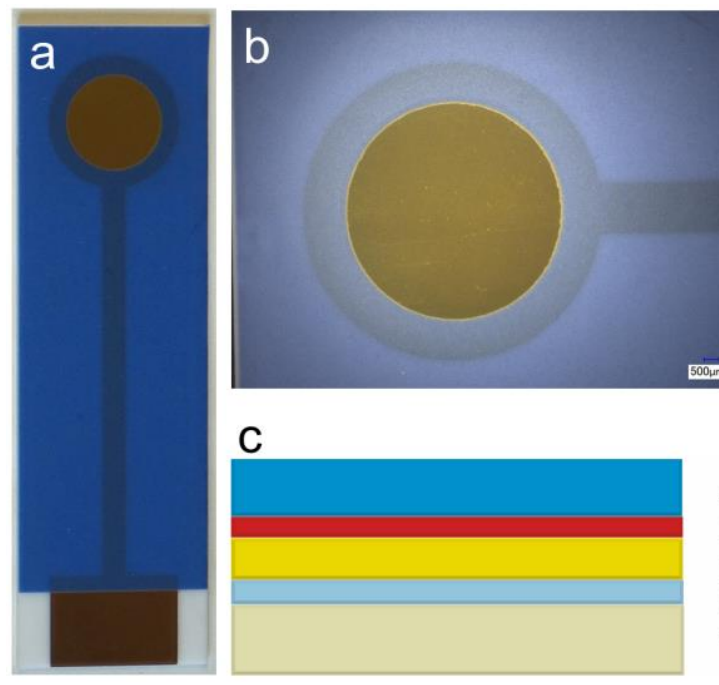

sensitive glass

interlayer $\left(\mathrm{Ti}_{x} \mathrm{O}_{\mathrm{y}}, \mathrm{Au}_{2} \mathrm{O}_{3}\right)$

pick-up electrode $(\mathrm{Au})$

dielectric glaze paste

alumina substrate

Figure 2. (a) Overall arrangement of structure 1. (b) Layout of the masked pick up electrode.

(c) Layer structure

For the thermal simulations, the structures were equipped with a platinum heater, which was designed for temperatures up to $350{ }^{\circ} \mathrm{C}$. The temperature control takes place by the evaluation of the resistance of a printed platinum conducting path. Furthermore, the discharge electrodes were executed as interdigital structures, according to Figure 3. Thus, impedance spectra and polarization experiments can be realized at different constant temperatures.

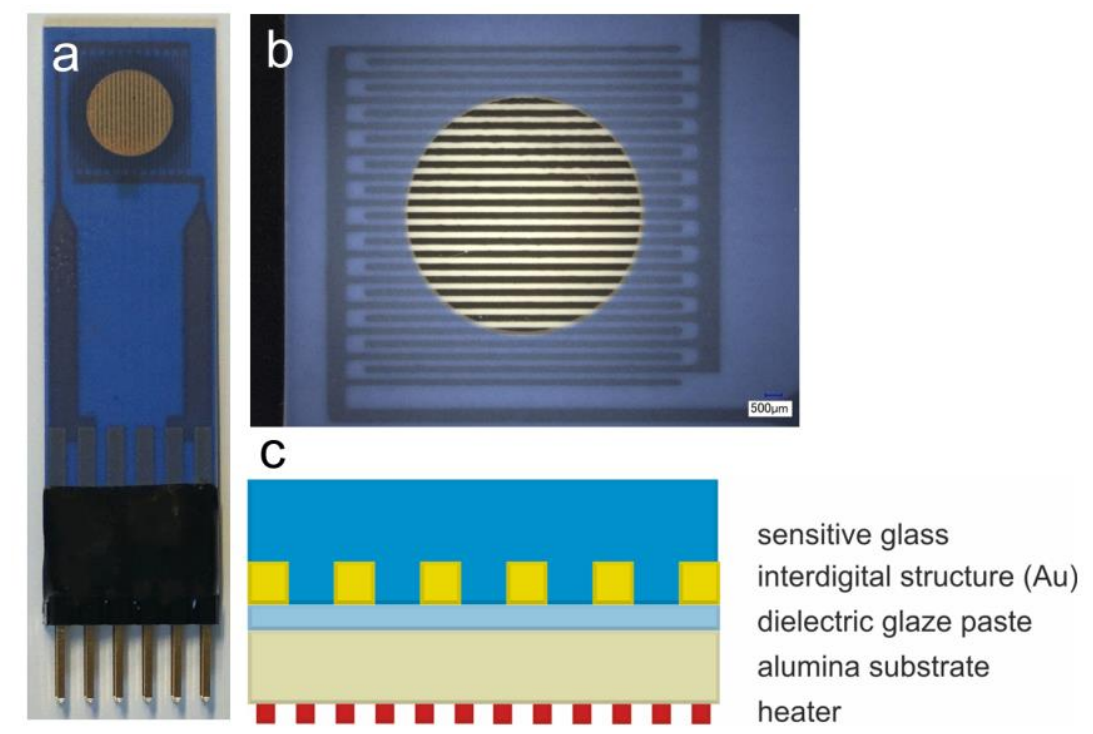

Figure 3. (a) Overall arrangement of structure 2. (b) Layout of the masked interdigital electrodes. (c) Layer structure 


\section{Preparation of sensitive glass layers by means of pulsed laser ablation}

The thin functional sensor layers were prepared by screen printing technique, sputtering methods and pulsed laser deposition (PLD) [17]. For this purpose, the combined coating system "CREAMET 500 PLD S2" with target carousel, substrate changer and mask changer was used. In this system, a sputtering system is also implemented for the deposition of intermediate layers $\left(\mathrm{Ti}^{\mathrm{T}} / \mathrm{Ti}_{\mathrm{x}} \mathrm{O}_{\mathrm{y}}\right)$ on the conductive path. Pre-cleaning by means of plasma is also possible in the sputtering chamber. With integrated substrate handler and mask change system 2 sputter targets, 6 PLD targets and altogether 5 changeable masks can be used and combined for the process. In order to ensure reproducible conditions during the processes, the laser entrance window is periodically cleaned by means of an ion source and the laser power is determined before and after a deposition process.

The deposition of the amorphous sensitive glass layers takes place with pulsed laser ablation (PLD) using an excimer laser COMPex Pro 110 (Coherent Inc., Santa Clara (USA)). It operates at a wavelength of $248 \mathrm{~nm}(\mathrm{KrF})$ and with a maximum pulse energy of $400 \mathrm{~mJ}$. The pulse length is a maximum pulse rate of $100 \mathrm{~Hz}, 20 \mathrm{~ns}$. Thus, a fluence of $5.3 \mathrm{~J} / \mathrm{cm}^{2}$ at $320 \mathrm{~mJ}$ is possible.

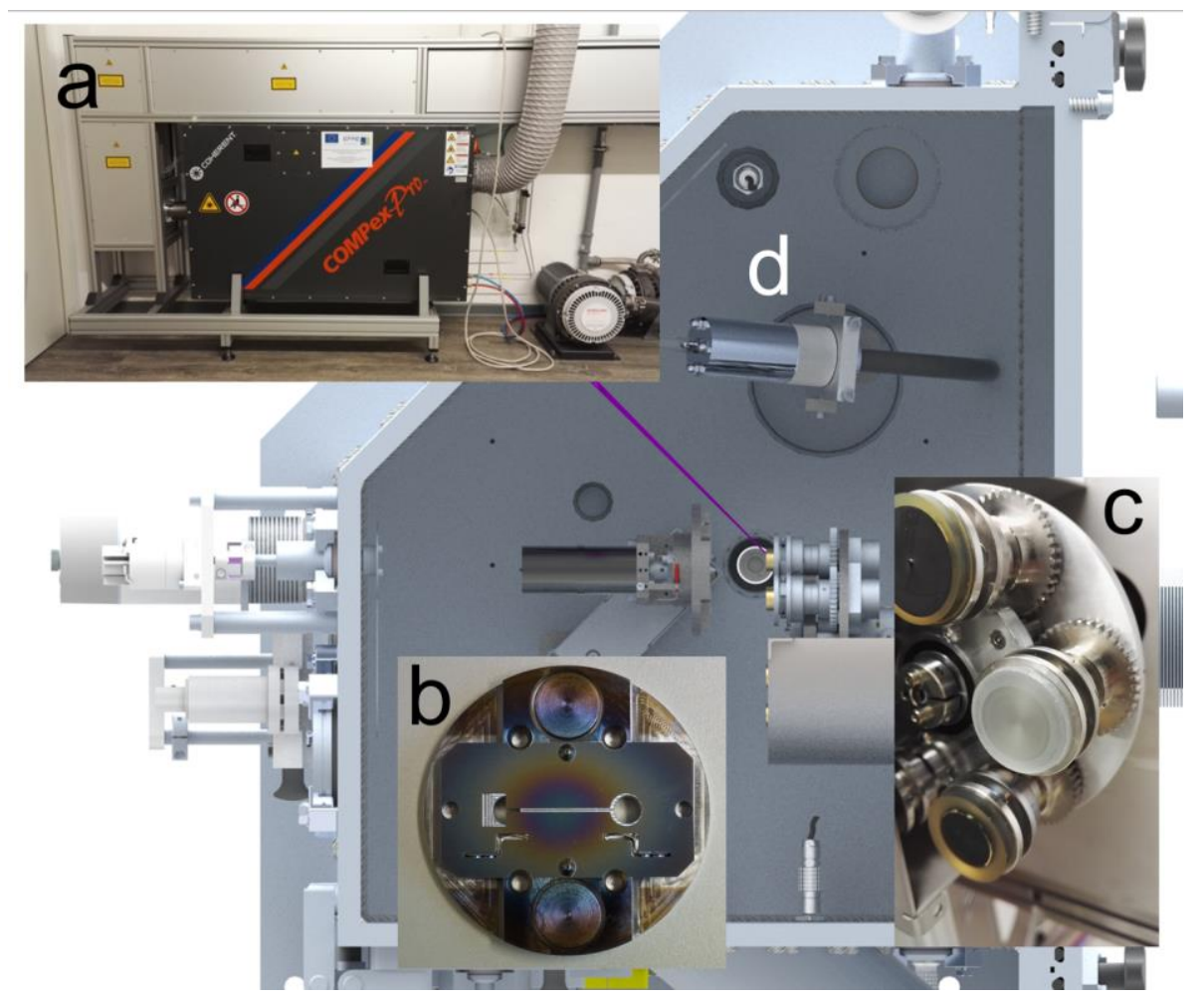

Figure 4. Optical beam path in the PLD chamber (a) Excimer laser COMPex Pro 110.

(b) Substrate holder with mask changer. (c) Target carousel. (d) Ion source

\section{Results and discussion}

In addition to the analytical methods (especially SEM, XRD, XPS and $\mu$-XRF) demonstrated elsewhere [18], the characterization of the properties of glass membranes was supplemented by electrochemical measurements.

\section{Electrochemical impedance spectroscopy}

When transferring classical electrochemical sensor principles into all-solid-state embodiments, special attention is directed to the internal interface transitions. On one hand, a liquid inner filling with a buffer effect must be replaced by a solid direct contact and, on the other hand, a reproducible potential at the interface between an ion-conducting layer and an electron-conducting lead should 
be ensured. The use of intermediate layers has a decisive influence on the potential formation and the drift behavior. In the present work, the conductive path made of gold was electrochemically oxidized and a semi-conductive oxide layer of $\mathrm{Ti}_{x} \mathrm{O}_{\mathrm{y}}$ was implemented. These two processes were executed with the aim of achieving a better electrical transition between ion-conducting glass and electron-conducting precious metal. The investigations were carried out by means of a geometrically stable and reproducible three-electrode arrangement with a glass membrane electrode as measuring electrode. The reference electrode was a $\mathrm{KCl}$-saturated silver/silver chloride electrode, and the counter electrode was a platinum sheet.

An appropriate tool for the characterization of glass based thin film systems is the recording of electrochemical impedance spectra. The impedance spectra (Figure 5) show an increased resistance in the layer structure $\mathrm{Au} / \mathrm{Au}_{\mathrm{x}} \mathrm{O}_{\mathrm{y}} / \mathrm{pH}$-glass. Then again, inserting of $\mathrm{Ti}_{\mathrm{x}} \mathrm{O}_{\mathrm{y}}$ semiconductive interlayers leads to a significant reduction in the total resistance of the measurement chain towards noninterlayer arrangements.

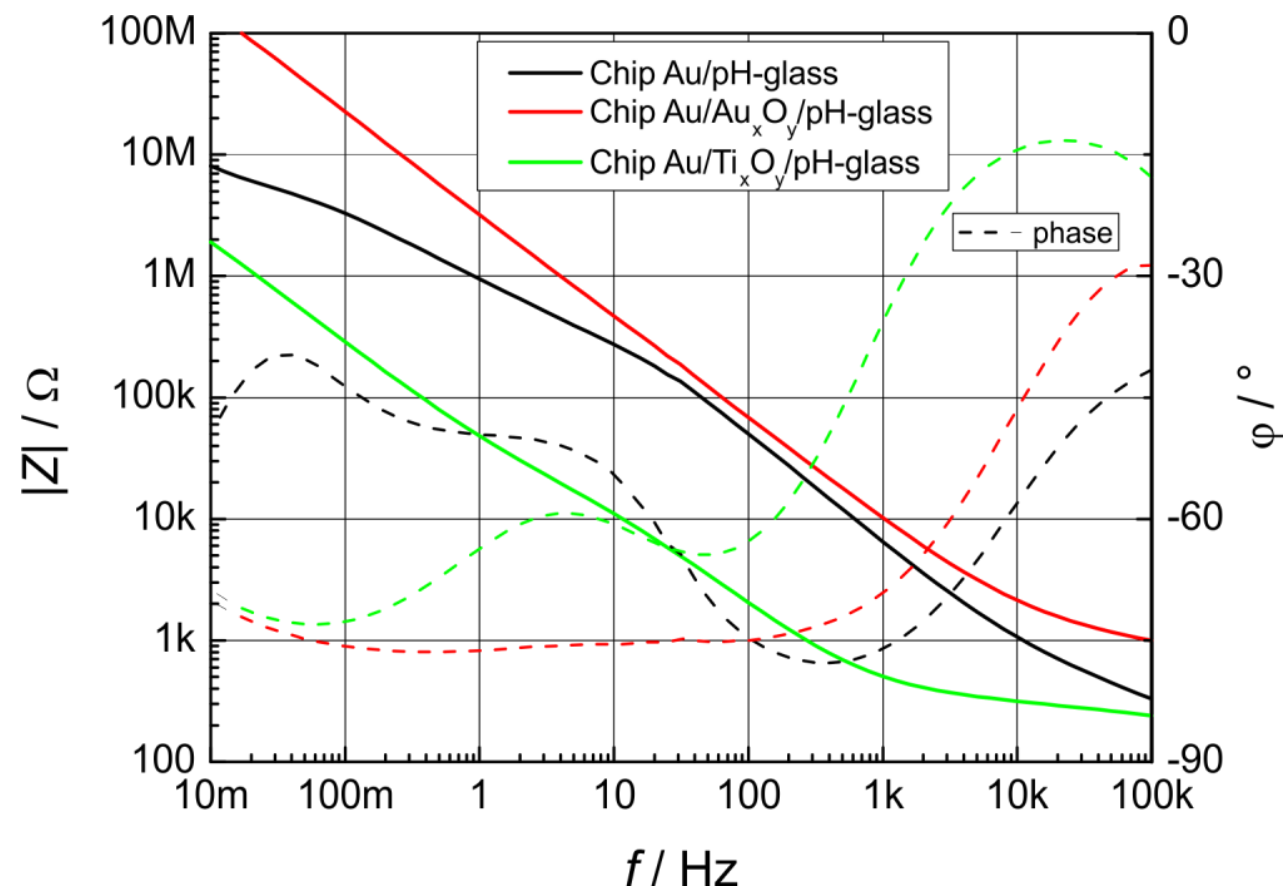

Figure 5. Electrochemical impedance spectra of gold-based electrodes covered with $\mathrm{pH}$-glass (with and without interlayers) - measured with three-electrode system consisting of working electrode, platinum electrode as counter electrode and $\mathrm{Ag} / \mathrm{AgCl} / \mathrm{KCl}$ sat.-reference electrode

The resulting spectra demonstrate that the total conductivity of all-solid-state configuration can be adjusted by chemical composition of the interlayers. Therefore, new sensitive glasses with higher resistance can be used for innovative sensor applications. In this context, it was interesting to find out, that glasses tending to segregation can effectively transferred with vacuum coating processes So, the fabrication of completely glass-based Lab-on-chip systems is conceivable.

\section{Running-in behavior and potential stability}

Electrode properties like running-in time, response time and sensor drift are particularly important for future users in terms of sensor acceptance. Again, not only the sensitive membranes but also the internal intermediate layers have direct influence on these properties.

In Figure $6 a$ it is shown, that the potential drift and the running-in behavior of the system $\mathrm{Au} / \mathrm{Ti}_{\mathrm{x}} \mathrm{O}_{\mathrm{y}} / \mathrm{pH}$-glass is smaller than the system Au/pH-glass. According to Figure $6 \mathrm{~b}$, the electrode 
signals of system $\mathrm{Au} / \mathrm{Ti}_{\mathrm{x}} \mathrm{O}_{\mathrm{y}} / \mathrm{pH}$-glass show smaller deviations in buffer solution of $\mathrm{pH} 6.86$, measured vs. $\mathrm{KCl}$-saturated silver/silver chloride reference electrode.
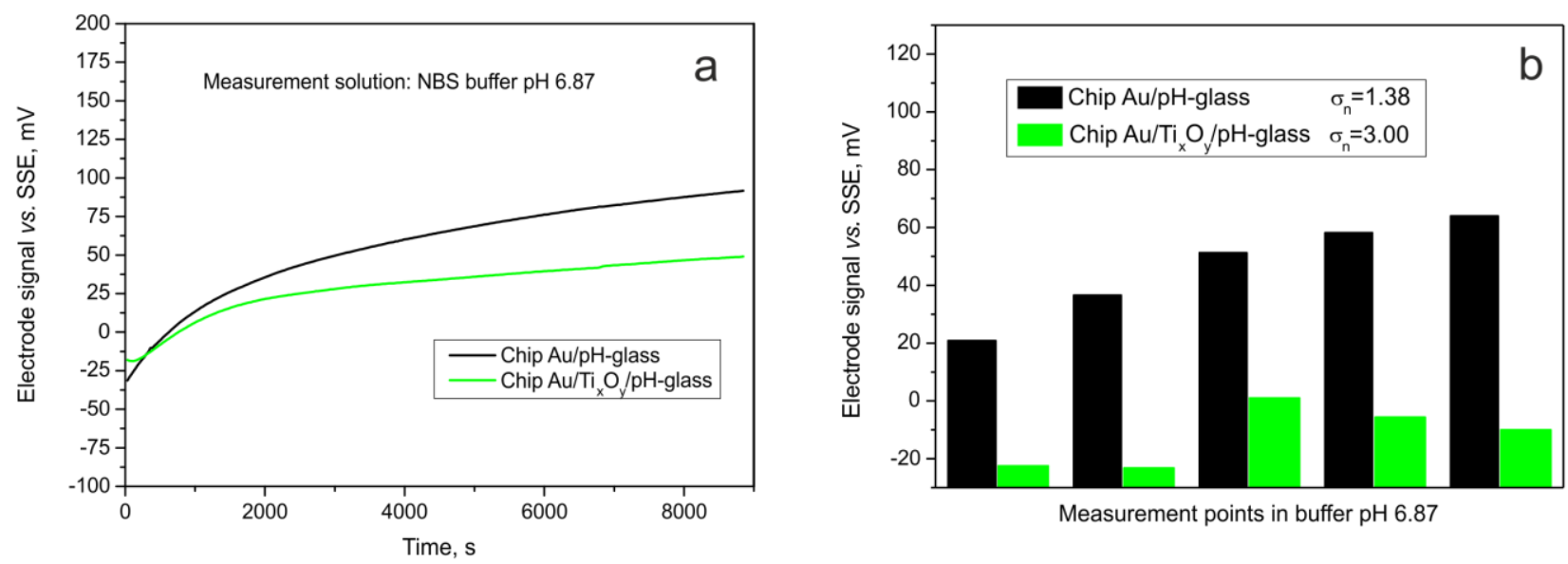

Figure 6. Influence of intermediate layers. (a) Running-in behaviors. (b) Stability of the open circuit voltage during several consecutive measurements in buffer solution $\mathrm{pH} 6.87$

\section{Sensor response and sensor slope}

Similarly, the sensitive membrane and conduction transfer layers also influence the other sensor properties. By deliberately influencing material compositions and sensor design, these sensor properties can be adjusted in a wide range. The direct changes in sensor response behavior and sensor slope are shown in Figure 7. Although the response times are similar, with the same $\mathrm{pH}$-sensitive membrane, only by using a semiconductive interlayer, the sensor slopes have been reduced by about $50 \%$. This makes it possible to develop inert glass-based reference electrodes with an extremely stable potential and low temperature drift.
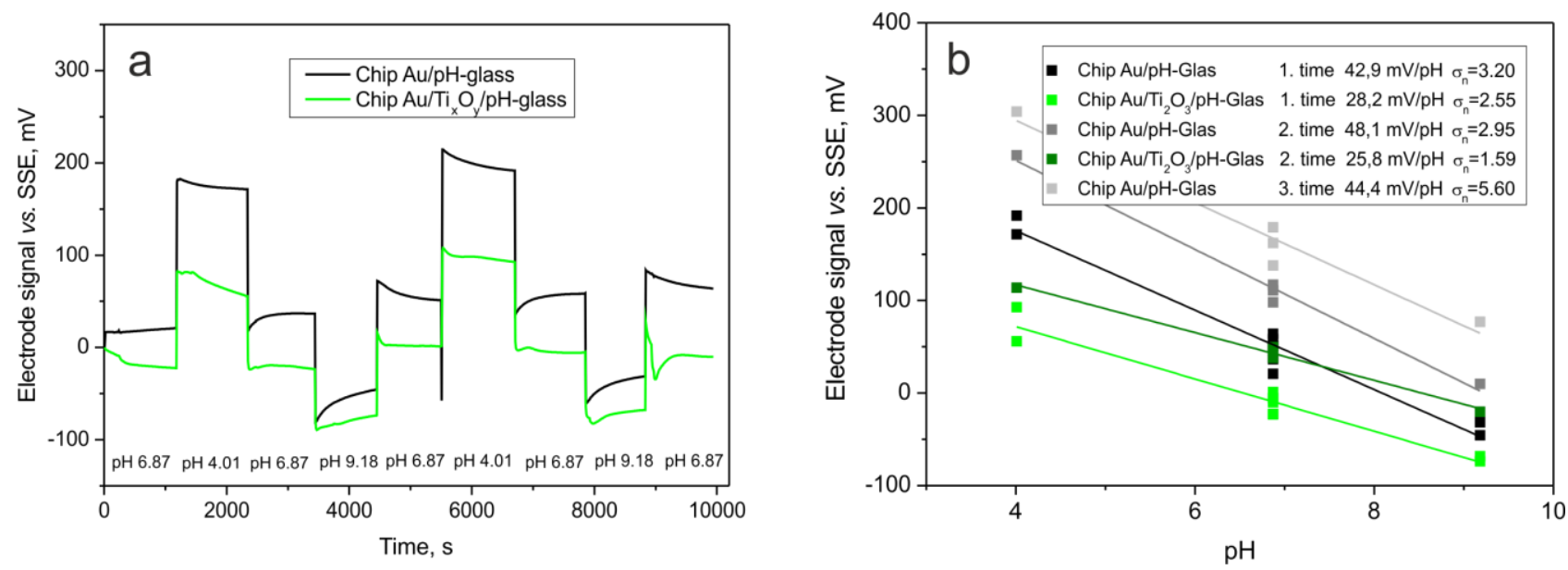

Figure 7. (a) Sensor response of Au-electrodes covered with $\mathrm{pH}$ glass membranes without and with $T_{x} O_{y}$-interlayer. (b) Sensor slope caused by change of sensor behaviors during repeated measurements in different $\mathrm{pH}$-solutions

Thermally stimulated currents (TSC)

In addition to usual analytical and electrochemical investigations, thermally stimulated characterizations of layer structures were carried out with the help of thermally stimulated currents (TSC) method. Hereby, it is explicitly possible to deduce the integral total mobility of charge carriers, 
in particular of alkali and alkaline earth ions. This is important for the development of highly conductive ion-sensitive glasses.

Figure 8a shows the cyclic voltammograms recorded on a Li-containing glass as a function of temperature deliver information about the ion mobility in sensitive glasses. The cyclic voltammograms at $250^{\circ} \mathrm{C}$ of glasses with charge carriers having different ionic radii are presented in Figure 8b. Glasses with small ions (e.g. Li-red curve) show higher currents than glasses with larger ones (e.g. La, Nd-blue curve). With this method, the total conductivities of glasses with different composition can be determined at variable temperatures.
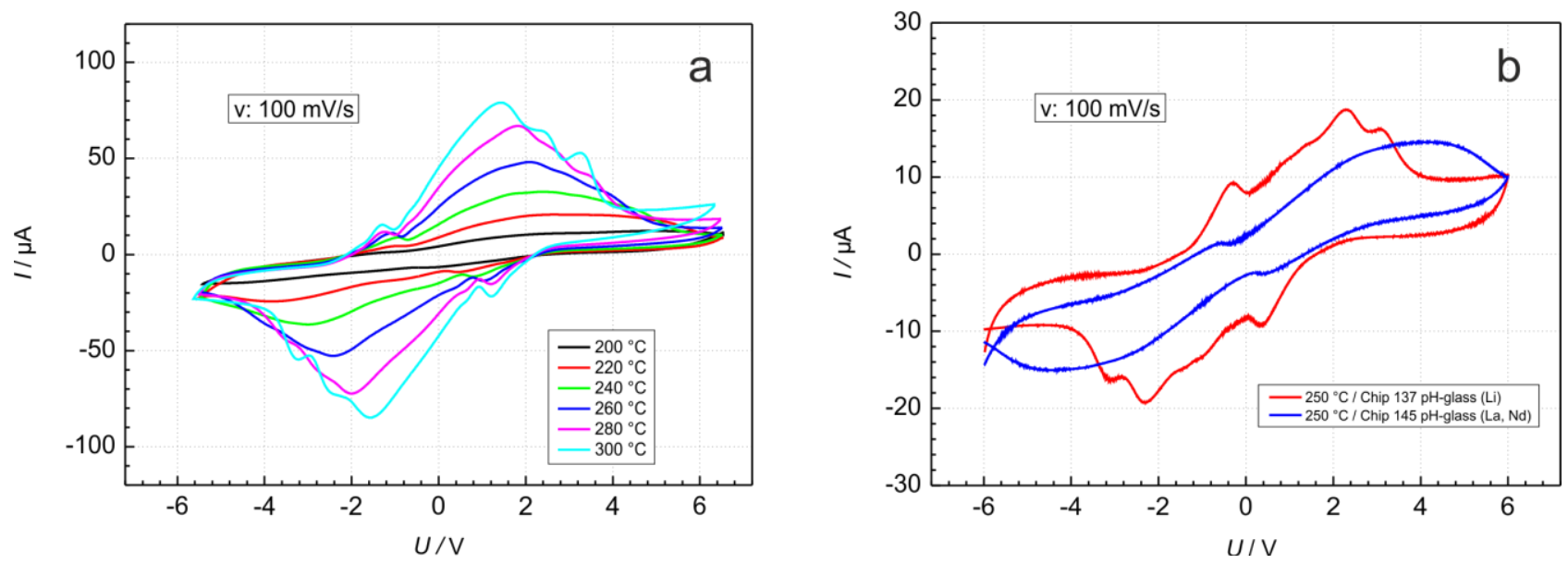

Figure 8. Cyclic voltammograms recorded on sensitive glass layers. (a) Variation of the temperature with integrated heater using Li-glass (glass 137). (b) Influence of glass conductivity by ion size and increased temperature (Li-containing glass/red, La, Nd-containing glass/blue).

\section{Conclusions}

There is a growing interest in all-solid-state sensors for various applications in process measurement technology, microreactor technology and environmental technology. That's why fundamental investigations into the development of sensitive membranes and on the mechanism of the behavior of the intermediate layers are necessary.

It has been shown that the PLD deposition process makes it possible to create homogeneous, amorphous glass structures. Their electrochemical sensor behavior can be described with the NERNST-equation. With the use of semiconducting intermediate layers, such as e.g. $\mathrm{Ti}_{x} \mathrm{O}_{y}$, it is possible to significantly reduce the total resistance of the device. This makes it possible to use the highimpedance, sensitive glasses for planar arrangements, such as lab-on-chip systems. It was demonstrated that glasses tending to segregation and therefore being unprocessable for the glassblower can be amorphously deposited in good quality by using vacuum technology (PLD).

Various integral electrochemical methods (electrochemical impedance spectroscopy, thermally stimulated currents) were applied extensively to characterize even very thin layers. The targeted selection of glass compositions for sensitive membranes and the use of suitable intermediate layers allow the manipulation of sensor properties in such a way that it will also be possible to generate insensitive reference systems in the future. Thus, all-solid-state measuring chains - working in the differential method according to Lübber's [11] - are conceivable as planar arrangement, in a miniaturized design without damaging discharge of liquids. 
Acknowledgements: Das Kurt-Schwabe-Institut für Mess- und Sensortechnik e.V. Meinsberg wird mitfinanziert durch Steuermittel auf der Grundlage des vom Sächsischen Landtag beschlossenen Haushaltes.

\section{References}

[1] K. Schwabe, pH measurement, Theodor Steinkopff, Dresden, D, 1976, pp. 120-186.

[2] G. Eisenman, Glass electrodes for hydrogen and other cations, Marcel Dekker, NY, 1967.

[3] R. E. Belford, R. G. Kelly, A. E. Owen, Thick film devices in chemical sensors, E. Edmonds, Ed., Blackie and Sons, London, 1988, pp. 236-255.

[4] V. G. Vlasov, Fresenius Z. Anal. Chem. 335 (1989) 92-99.

[5] T. Bachmann, J. Spindler, F. Gerlach, W. Vonau, J. Univ. of Appl. Sci. Mittweida 6 (2006) 3-7.

[6] T. Bachmann, J. Spindler, F. Gerlach, W. Vonau, Patent DE 102005059680 A1 (2005).

[7] M. Frumar, B. Frumarova, P. Nemec, T. Wagner, J. Jedelsky, M. Hrdlicka, Journal of Non-Crystalline Solids 352 (2006) 544-561.

[8] J.P. Klook, L. Moreno, A. Bratov, S. Huachupoma, J. Xu, T. Wagner, T. Yoshinobu, Y. Ermolenko, Y.G. Vlasov, M.J. Schöning, Sensors and Actuators B, 118 (2006) pp. 149-155.

[9] O.G. Pompilian, G. Dascalu, J. Mihaila, S. Gurlui, M. Olivier, P. Nemec, V. Nazabal, N. Cimpoesu, C. Focsa, Applied Physics A, 117 (2014) 197-205.

[10] W. Vonau, M. Decker, J. Zosel, K. Ahlborn, F. Gerlach, S. Weißmantel, Sensors \& Transducers, 193(10) (2015) pp. 93-99.

[11] D.W. Lübbers, The Science of Nature 49(21) (1962) 493.

[12] Pfaudler Emaillierte pH-Sonden, https://www.pfaudler-messtechnik.de/de/products/liquidanalysis/ph-measurement/ (2019).

[13] pH-Sensor InPro4850i, https://www.mt.com/de/de/home/library/technical-reports/processanalytics/pH InPro4850i (2019).

[14] Analoger glasfreier pH-Sensor Tophit CPS471, https://portal.endress.com/wa001/dla/5000315/8755/000/05/TI283CEN US 0510.pdf (2019).

[15] L. Florian, B. Savu, F. Sima, I.N. Mihailescu, D. Tanasković, D. Janacković, Digest Journal of Nanomaterials and Biostructures 2(3) (2007) pp. 285-291.

[16] W. Vonau, F. Gerlach, U. Enseleit, J. Spindler, T. Bachmann, Journal of Solid State Electrochemistry 13 (2009) 91-98.

[17] F. Gerlach, K. Ahlborn, S. Sachse, W. Vonau, Sensor \& Transducers 184 (2015) 39-44.

[18] K. Ahlborn, F. Gerlach, W. Vonau, International Journal on Advances in Systems and Measurements 10(1\&2) (2017) 56-63.

(C)2019 by the authors; licensee IAPC, Zagreb, Croatia. This article is an open-access article distributed under the terms and conditions of the Creative Commons Attribution license (http://creativecommons. org/licenses/by/4.0/) 